\title{
Study on Compound Biofilm Formation on the surface of Iron Oxide Coated Sands and Its Removal Efficiency to Ammonia-Nitrogen
}

\author{
Li Dongmei \\ Guangdong University of Technology \\ Guangzhou, China, Phone
}

Huang mingzhu

Foshan Water Group

Foshan, China, Phone

\author{
Liu Bei \\ Guangdong University of Technology \\ Guangzhou, China, Phone
}

Lin xianzeng

Foshan Water Group

Foshan, China, Phone

\author{
Huang Taifu \\ Guangdong University of Technology \\ Guangzhou, China, Phone \\ Ye tingjin \\ Foshan Water Group \\ Foshan, China, Phone
}

\begin{abstract}
By using a homemade Iron Oxide Coated Sands filtration medium (for short: IOCS), start-up behaviors of compound biofilm formation on the surface of IOCS were investigated with the help of a series of experiments, and its removal efficiencies to Ammonia-Nitrogen contained in micropollution raw water were analyzed as well. The differences of removal efficiency between raw quartz sands (for short: RQS) coated biofilm and bio-IOCS were discussed. Surface morphologic properties of two kinds of bio-sands were analyzed too. The results are shown as follows: The biomass of Bio- RQS, and Bio-IOCS are $15.46 \mathrm{nmolP} /(\mathrm{g}$ sand) and $13.79 \mathrm{nmolP} /(\mathrm{g}$ sand) at the initial stage of biofilm formation, $18.75 \mathrm{nmolP} /(\mathrm{g}$ sand) and $20.09 \mathrm{nmolP} /(\mathrm{g}$ sand) at the stable stage. When the concentration of Ammonia-Nitrogen is in the range of $1.0 \mathrm{mg} / \mathrm{L}$ to $2.0 \mathrm{mg} / \mathrm{L}$, removal rate of Bio- RQS and Bio-IOCS is $92 \%, 95 \%$ separately at the initial stage, and it is $60 \%, 80 \%$ respectively at the stable stage. If concentration of Ammonia-Nitrogen varies from 0.5 $\mathrm{mg} / \mathrm{L}$ to $4.0 \mathrm{mg} / \mathrm{L}$, removal rate of Bio- RQS increases from $60 \%$ to $80 \%$, and Bio-IOCS from $70 \%$ to $95 \%$. The surface morphologic properties of the two filters are quite different before and after filtration and its biofilm formation. The surface pore sizes become smaller, surface appearances are rougher and its porosity is high. Surface morphologic characteristics of BioIOCS are changed more markedly than those of IOCS and bioRQS.
\end{abstract}

Keywords-Iron Oxide Coated Sand; Bio-Raw Quartz Sands; Bio-Iron Oxide Coated Sands; Compound Formation; AmmoniaNitrogen; Removal Efficiency; Morphologic Properties

\section{INTRODUCTION}

It is one of tough problems that how to remove AmmoniaNitrogen efficiently and inexpensively from micro-pollution raw water based on conventional water treatment processes. Many waterworks feel confused about the safety of byproducts after adding chlorine. The removal efficiency of conventional processes-"coagulation-sedimentation-filtration-disinfection" is weak for the treatment of dissolved Ammonia-Nitrogen [1].Biological method is one of the main ways to remove Ammonia-Nitrogen effectively [2]. At present, the study on

Natural Science Foundation of China (No 51108094),

Science and Technology Research Foundation of Guangdong Province (No 2010B030900001),

Hong Kong-Guangdong Breakthrough Projects in Key Areas (Foshan special: No 2010Z524). using biological method to remove Ammonia-Nitrogen mainly focused on attached biofilm of the following media surface: raw quartz sands, zeolites, and activated carbons, and etc. However, how to make microorganisms closely adhere to the surface of filter media is still one of the problems which should be solved urgently [3-9]. After coated modifier on the surface of raw quartz sands (for short: RQS), Iron Oxide Coated Sands (for short: IOCS) are prepared with large specific surface area, strong ion exchange ability and a large ammonium adsorption capacity in water [10]. IOCS is of low production cost and simple operation. When IOCS has been covered with a layer of stable biofilm, we call it Biological Iron Oxide Coated Sands Filter (for short: Bio-IOCS). Bio-IOCS combines the nitrification of microorganisms with adsorption exchange of IOCS, which can achieve stable, effective efficiency to remove Ammonia-Nitrogen, The removal rate of Bio-IOCS to Ammonia-Nitrogen is compared with that of Bio-RQS, which can lead to determination the optimum biofilm formation conditions and removal efficiency of Ammonia-Nitrogen. Hence, it can be helpful for elimination of Ammonia-Nitrogen contained in micro-pollution raw water.

\section{Methods AND MAterials}

\section{A. Materials}

Experimental filter Columns are made of organic glasses, 7 $\mathrm{cm}$ in diameter. The filter media are RQS and IOCS, whose size is in the range of $0.6 \mathrm{~mm}-1.2 \mathrm{~mm}$. The depth of filter media layer is $40 \mathrm{~cm}$ high, Water is downward flow. micropollution raw water for filtration comes from effluent of sedimentation tank, whose turbidity is $1.0 \pm 0.3 \mathrm{NTU}$, an average $\mathrm{pH}$ is $7.4 \pm 0.4$, concentration of $\mathrm{COD}_{\mathrm{Mn}}$ is $1.5 \mathrm{mg} / \mathrm{L}$ $\pm 0.4 \mathrm{mg} / \mathrm{L}$, Ammonia-Nitrogen concentration varies from $1 \mathrm{mg} / \mathrm{L}$ to $2 \mathrm{mg} / \mathrm{L}$.

\section{B. Experimental Procedures}

Combined with the characteristics of activated sludge inoculation method and those of natural bio-film inoculation 
method, compound biofilm formation technology was used in experiments: to aerate activated sludge inoculation suspension , increase flowrate gradually, reduce sludge volume little by little. Here, the period of continuous aeration with peristaltic pump is 10 days, whose aeration flowrate is $4.6 \mathrm{~L} / \mathrm{h}$. Then backwashing with air and water alternately goes on 6 mins, which runs once every 2 days. The strength of backwashing with air and water is: $\mathrm{q}_{\mathrm{air}}=6 \mathrm{~L} / \mathrm{m}^{2} \cdot \mathrm{s}, \mathrm{q}_{\text {water }}=5 \mathrm{~L} / \mathrm{m}^{2} \cdot \mathrm{s}$ respectively. When flowrate is low in $2 \mathrm{~L} / \mathrm{h}$, filtration keeps running for 10 days, then flowrate is changed from $2 \mathrm{~L} / \mathrm{h}$ to $5 \mathrm{~L} / \mathrm{h}$ without backwashing for another 15 days. Inoculation suspension is prepared with tap water, sludge and nutrition liquid. Here, tap water comes from NanZhou Waterworks, Guangzhou, Guangdong, China; sludge is from secondary sedimentation tank of LiJiao Sewage Plant, Guangzhou, Guangdong, China; nutrition liquid is the concoction of $\mathrm{C}, \mathrm{N}$ and $\mathrm{P}$, the proportion of $\mathrm{C}: \mathrm{N}: \mathrm{P}$ is 100:5:1; glucose serves for carbon source, $\mathrm{NH}_{4} \mathrm{Cl}$ for nitrogen source and potassium dihydrogen phosphate for phosporus source.

\section{1) Biomass Measurement}

At different biofilm formation stage, sands attached microorganisms are sampled from 3 different height in the filter column. Before sampling, water in filter is drained with flowrate $1.5 \mathrm{~m} / \mathrm{h}$. Put samples to $100 \mathrm{~mL}$ stoppered conical flask, add chloroform, methanol, and water, whose concentration ratio is $1: 1: 0.9$, to the flask one by one respectively. About 12 hours later, adding $5 \mathrm{~mL}$ water and $5 \mathrm{~mL}$ chloroform in this monophasic system to separate the phases, the total lipid fraction will be found in the chloroform phase which is in the lower layer of the flask after resting for another 12 hours, then sample $5 \mathrm{~mL}$ of chloroform phase with stoppered graded tube $10 \mathrm{~mL}$ in volume (for short: tube), put the tube into the water bath to evaporate to dryness. $0.8 \mathrm{~mL}$ of $5 \%$ potassium persulfate solution and distilled water are added to the tube to $10 \mathrm{~mL}$ scale, 30 minutes digestion in the high-pressure steam sterilization pot at $121^{\circ} \mathrm{C}$. Afterwards, add $0.2 \mathrm{~mL}$ of $10 \%$ ascorbic acid and shake uniform, continue adding another chemical- $0.4 \mathrm{~mL}$ molybdenum solution, mix fully, then keep it at room temperature for 15 minutes, measure the absorbance by using water as reference, $700 \mathrm{~nm}$ wavelength, $1 \mathrm{~mm}$ colorimetric cuvette. According to the results of biomass spectrophotometry, and the standard curve for conversion to $\mathrm{nmolP} / \mathrm{g}$ filter material, that is to said, $1 \mathrm{nmolP}$ is equal to the $\mathrm{e}$. coli (E.coli) the size of $10^{8}$ cells.

2) Experimental Setup

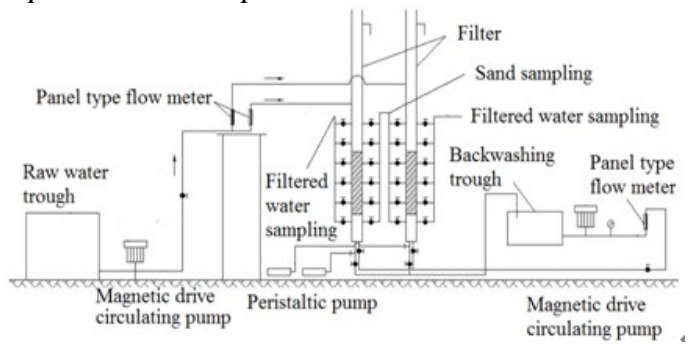

Fig.1 The flow chart of experimental setup

Raw water contained micropollutant - Ammonia-Nitrogen is sent to filter columns from the trough through pump. Filtration runs $12 \mathrm{~h}$ continuously without aeration every day, no flowrate at night. Water level above the surface of the filter columns approximately keeps $10 \mathrm{~cm}$ high. Filtration rate is $1.5 \mathrm{~m} / \mathrm{h}$ with dissolved oxygen $(\mathrm{DO}) \geqslant 3 \mathrm{mg} / \mathrm{L}$ throughout media layer. Water is at temperature $28 \pm 5^{\circ} \mathrm{C}$ and $\mathrm{pH} 7.4 \pm 0.4$ in the period of experiments. The test device is shown in Fig.1.

\section{3) Test Factors and Methods}

All experimental effluent samples were collected in $250 \mathrm{~mL}$ polypropylene bottles and then immediately analyzed after sampling.

- Residual concentration of ammonium nitrogen $\left(\mathrm{NH}_{4}^{+}\right.$$\mathrm{N})$ : A nitrate cellulose membrane filter, $0.45 \mu \mathrm{m}$ of pore size(Shanghai, China), was used to filter the undissolvable mixed liquor suspended solids (MLSS) that would affect the results of measurement. The absorbance of ammonium nitrogen $\left(\mathrm{NH}_{4}{ }^{+}-\mathrm{N}\right)$ were determined spectrophotometrically through the Nessler Reagent Method at an absorbance of 420nm using a T6 UV Spectrophotometer (Beijing, China), then residual concentration of ammonium nitrogen can be sure according to the concentration - absorbance standard curve.

- Removal rate: Removal efficiency of the responses was obtained using the following equation:

$\mathrm{NH}^{+}{ }_{4}-\mathrm{N}(\%)=\left(\mathrm{N}_{\mathrm{i}}-\mathrm{N}_{\mathrm{f}}\right) / \mathrm{N}_{\mathrm{i}} * 100$

Where $\mathrm{N}_{\mathrm{i}}, \mathrm{N}_{\mathrm{f}}$ is the initial concentration $(\mathrm{mg} / \mathrm{L})$ of $\mathrm{NH}_{4}^{+}-\mathrm{N}$, and the final concentrations respectively.

- Biomass: Biomass is determined with the help of T6 UV spectrophotometer (Beijing, China) through the Lipid Phosphorus Method at an absorbance of 720nm, in accordance with the standard curve.

- Surface morphologic characteristic: Surface morphologic characteristic was analyzed by using S3400N Scanning Electron Microscope (SEM) (Hitachi, Japan).

\section{RESULTS AND DisCUSSIONS}

\section{A. Comparison of Biomass attached on the surface of RQS and IOCS}

Biofilm compound formation was tested in two filtration columns which were equipped with RQS and IOCS. Biomass attached on RQS and IOCS at the initial stage and the stable stage are shown in Fig. 2.

The biomass of Bio-RQS and Bio-IOCS are 15.46 and $13.79 \mathrm{nmolP} /(\mathrm{g}$ sand) at the initial stage of biofilm formation, $18.75 \mathrm{nmolP} /(\mathrm{g}$ sand) and $20.09 \mathrm{nmolP} /(\mathrm{g}$ sand) at the stable stage respectively. The biomass of Bio-RQS slightly higher 


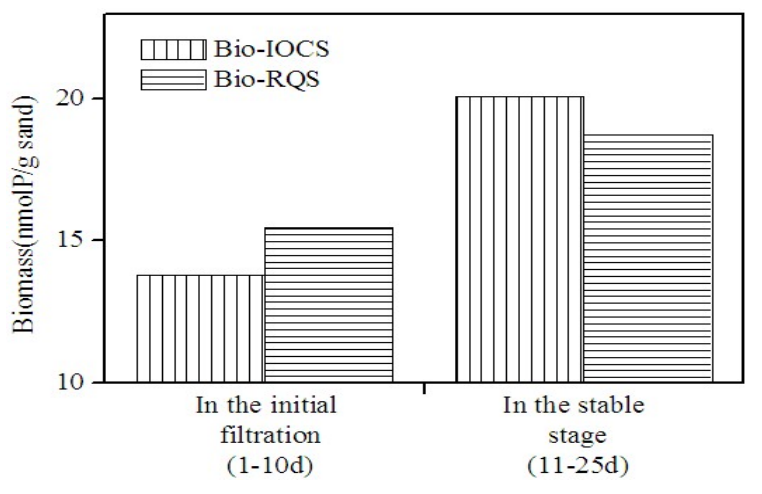

Fig.2 Analysis of biomass atteached on RQS and IOCS

than that of Bio-IOCS at the initial stage. However, at the stable stage, the biomass of Bio-RQW is lower than that of Bio-IOCS, which is caused by enormous specific surface area and strong adsorption capacity of IOCS, and the biomass attached on RQS is easier to strip off. But there is enough biomass attached on both two filter media, which can provide a stable operation with biological adsorption and interception filtration.

\section{B. Removal Efficiency of Bio-RQS and Bio-IOCS to Ammonia-Nitrogen}

At the initial stage, after microorganisms growth and attached on the surface of filter media for 7 days, the removal rate of two filters to Ammonia-Nitrogen keeps nearly stable (seen from Fig. 3). But the removal efficiency of Bio-IOCS to Ammonia-Nitrogen is slightly higher than that of Bio-RQS. The former is up to $95 \%$, the latter is $92 \%$. While the removal rate of IOCS and RQS to Ammonia-Nitrogen is lower before they are covered with microorganisms. The former is up to $20 \%$, the latter is $15 \%$.

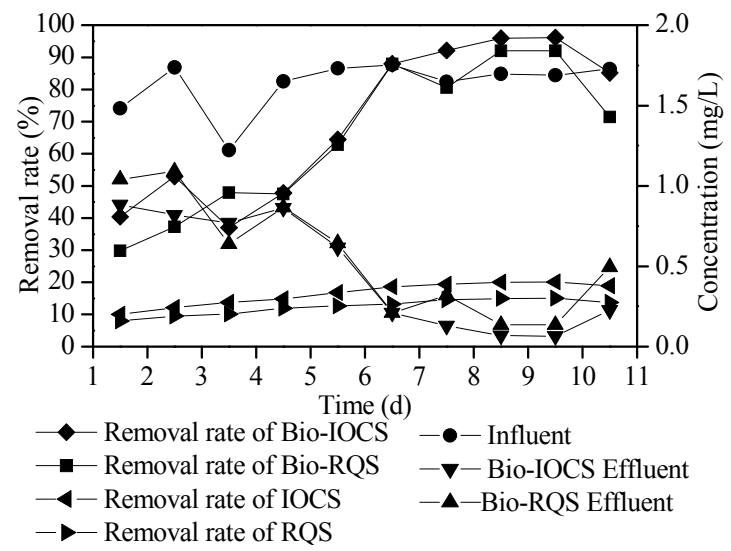

Fig.3 Variation of the removal rate of different filtration media to ammonia nitrogen with time at the initial stage of biofilm formation

When the concentration of Ammonia-Nitrogen varies from $1.0 \mathrm{mg} / \mathrm{L}$ to $2.0 \mathrm{mg} / \mathrm{L}$, the removal rate of Bio-RQS and BioIOCS corresponding increases from $92 \%$ to $95 \%$ at the initial stage of biofilm formation, and $60 \%$ to $80 \%$ at the stable stage (seen from Fig. 4). At the stable stage, the removal rate of
IOCS and RQS to Ammonia-Nitrogen is around 17.5\%, 15\% on average respectively. Because the adhesion ability of RQS is weak and biofilm is easy to strip off. The removal rate is unstable. However, IOCS has a large specific surface area and adsorption capacity, which makes a sufficient number of biomass tightly adsorbed. Under the action mechanisms of physical adsorption of IOCS and the bio-oxidation of microorganisms, ammonia nitrogen is degraded dynamically and continuously without backwashing at the stable stage.

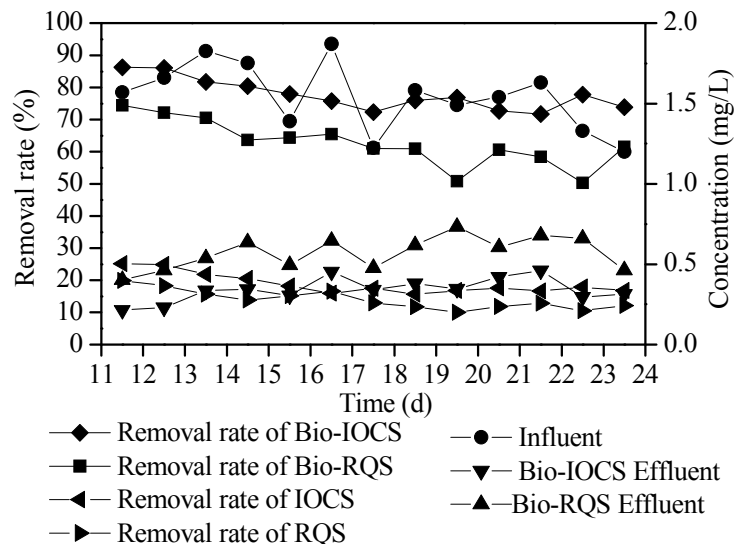

Fig.4 Variation of the removal rate of different filtration media to ammonia nitrogen with time in the stable stage

\section{Effects of Ammonia-Nitrogen Concentration on Removal Efficiency}

At the stable stage, when the concentration of AmmoniaNitrogen varies from $0.5 \mathrm{mg} / \mathrm{L}$ to $4.0 \mathrm{mg} / \mathrm{L}$, the comparison of the removal efficiency of Bio-RQS and Bio-IOCS is shown in Fig.5.

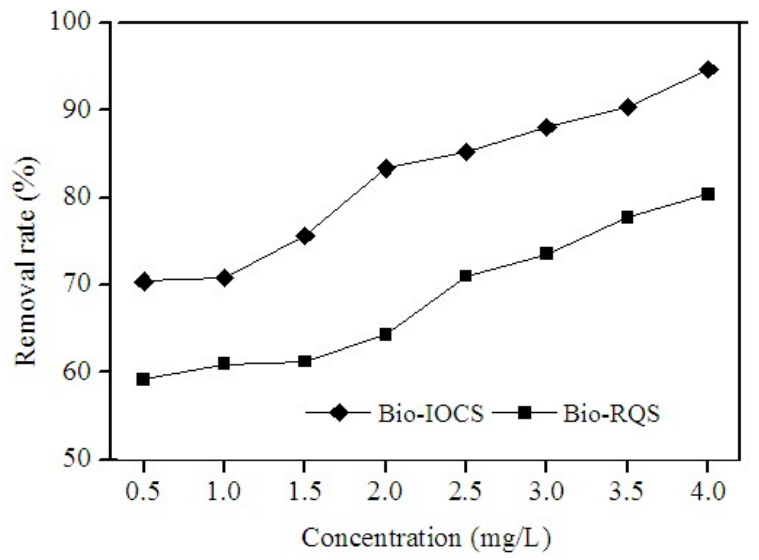

Fig.5 Comparison of removal rate of different concentrations ammonia nitrogen at the stable stage

Seen from Fig.5, the average removal rate of two kinds of bio-filtration goes up with the increasing concentration of Ammonia-Nitrogen. when concentration varies from $0.5 \mathrm{mg} / \mathrm{L}$ to $4.0 \mathrm{mg} / \mathrm{L}$, removal rate of Bio- RQS increases from $60 \%$ to $80 \%$, and Bio-IOCS from $70 \%$ to $95 \%$. The growth rate of biomass coated on IOCS is higher than that on RQS 
significantly. At the same Ammonia-Nitrogen concentrations, the removal rate of Bio-IOCS is obviously higher than that of Bio-RQS, for the large specific surface area of IOCS makes biofilm attached on the surface tightly and stably. Furthermore, nitrification bacteria in the biofilm have high activity and adsorption capacity.

\section{Surface Morphological Characteristics of Bio-IOCS and Bio-RQS}

As above mentioned, to remove Ammonia-Nitrogen from micro-pollution raw water, Bio-IOCS has noticeable advantages compared with Bio-RQS. The main reason is that surface morphological characteristics are different markedly (shown in Fig.6A-Fig.6D). The surface of IOCS presents more rough and porous structures (seen from Fig.6A), specific surface area significantly increases, covered with blocks of $\mathrm{Fe}_{3} \mathrm{O}_{4}$ (magnetite) oxide,. Improving the isoelectric point of filter media will lead to microorganisms firmly attached to the surface (seen from Fig.6B). Under the action of strong adsorption and interception capability of IOCS, and its biodegradation, the removal rate of Bio-IOCS to AmmoniaNitrogen goes up notably. While the surface appearance of RQS is relatively smooth (shown in Fig.6C), it has small specific surface area and weak adsorption capacity. When microorganisms attached on it (seen from Fig.6D), although surface roughness is enhanced, adhesion strength of the creatures on its surface is weak, lower biomass on RQS than on that of IOCS, which results in low removal efficiency.

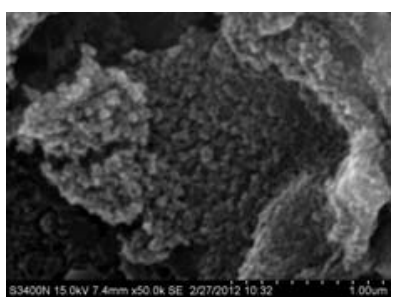

A-IOCS

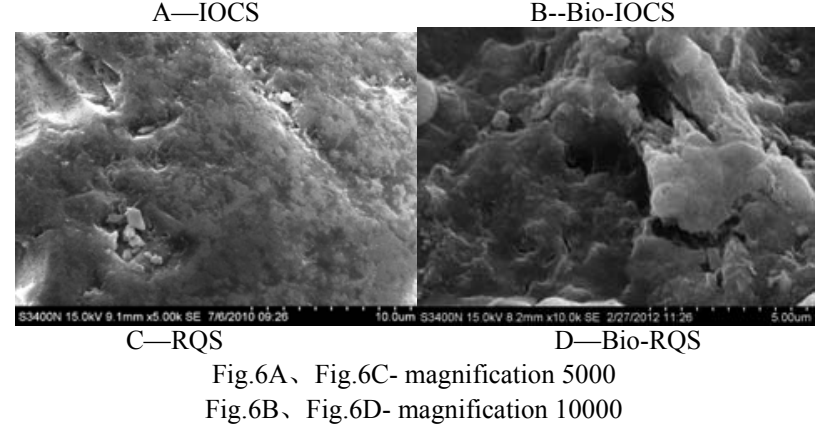

Fig.6 The SEM photos of different filtration media

\section{CONCLUSIONS}

a) The surface of IOCS and RQS can be successfully covered with microorganisms by compound biofilm formation technology. The removal efficiency of Bio-IOCS and BioRQS to Ammonia-Nitrogen is roughly $90 \%$, which is higher than that of IOCS and RQS.

b) Both RQS and IOCS can be covered with enough biomass to ensure the stable operation of the filters. Biomass on the surface of Bio-IOCS is $1.34 \mathrm{nmolP} /(\mathrm{g}$ sand), which is higher than that of Bio-RQS at stable formation stage.

c) The removal rate of Bio-IOCS and Bio-RQS to Ammonia-Nitrogen is nearly the same, around 95\%, 92\% respectively at the initial stage. The removal rate of Bio-IOCS to Ammonia-Nitrogen is approximately $20 \%$ higher than that of Bio-RQS. and the removal efficiency of Bio-IOCS can stabilize at $80 \%$ at the stable stage.

d) At the stable stage, when Ammonia-Nitrogen concentration varies from $0.5 \mathrm{mg} / \mathrm{L}$ to $4 \mathrm{mg} / \mathrm{L}$, removal rate of bio-IOCS to Ammonia-Nitrogen is significantly higher than that of bio-RQS: removal rate increases from $70 \%$ to $95 \%$ for the former; $60 \%$ to $80 \%$ for the latter. At the same concentration of Ammonia-Nitrogen, removal rate of bioIOCS is about $20 \%$ higher than that of bio-RQS.

e) The surface morphological characteristics of IOCS has more rough and porous structure, specific surface area significantly increases and microorganisms tightly attached to the surface. The attached biofilm makes the roughness and porosity on the surface of filter media increasing further, thus, it has stronger physical adsorption abilities and biodegradation and bio-oxidation capacities.

\section{REFERENCES}

[1] Wang Zhansheng, Liu Wenjun, "Micro-pollution Treatment Technology for Drinking Water," China Architecture \& Building Press, 1999.(in Chinese)

[2] The Peoples's Republic of China Ministry of Health,the National Standardization Management Committee, "Drinking water health standards," GB 5749-2006, China. (in Chinese)

[3] Wu Fuping, Qi Haiying, "Study of Biofilm Cultivation Process in the Treatment of Slightly Polluted Cellar Rainwater by Activated CarbonQuartz Biological Filtration", Technology of Water Treatment, vol. 37, pp. 70-72, 86, 2011. (in Chinese)

[4] Yang Jingwen, Jiang Shuangying, "Research on Start-up of Biological Aerated Filter in Low Temperature," Technology of Water Treatment, vol. 37, pp. 112-115, 2011. (in Chinese).

[5] You Zhilei, Chen Linghu, "Evaluation on Initial Operation Effect of Biological Activated Carbon Filter," China Water \& Wastewater, vol. 25, pp. 93-96, 2009. (in Chinese)

[6] Li Simin, Su Chengyuan," Experimental Study on Different Biofilm Formation Methods in Biological Sand Filter," China Water \& Wastewater, vol. 23, pp. 60-63, 2007. (in Chinese).

[7] Liu Bing, Wei Bo, "Impact of temperature and backwashing on biofilter in drinking water process", China Water \& Wastewater, vol. 35, pp. 29-33, 2009.

[8] David R.Simpson. "Biofilm processes in biologically active carbon water purification,” Water Research, vol. 42, pp. 2839-2848, 2008.

[9] Chunping Yang, Hong Chen, "Biomass accumulation and control strategies in gas biofiltration," BiotechnologyAdvances, vol. 28, pp. 531-540, 2010.

[10] Li Dongmei, Huang Hui, "Study on the Optimum Preparation Process Conditions of Iron Oxide Coated Sand ," CDCIEM, pp.1823-1826, 2011 International Conference on Computer Distributed Control and Intelligent Environmental Monitoring, 2011. 